\title{
28 Research Square \\ Predicting diagnosis, prognosis of hepatocellular carcinoma based on iron activity
}

Bufu Tang

Jinyu Zhu

Jie Li

Kai Fan

Yang Gao

Shimiao Cheng

Chunli Kong

Liyun Zheng

Fazong Wu

Qiaoyou Weng

Chenying Lu

Jiansong Ji

\section{Video Byte}

Keywords: Cell Communication and Signaling, ferroptosis, hepatocellular carcinoma, HCC, TMB, immune microenvironment, prognosis, diagnosis, iron, metabolism, liver, tumor growth, erastin, immunotherapy, differentially expressed genes, DEGs, LASSO, nomogram, TCGA, The Cancer Genome Atlas

Posted Date: November 12th, 2020

DOI: https://doi.org/10.21203/rs.3.rs-107415/v1

License: (c) (1) This work is licensed under a Creative Commons Attribution 4.0 International License. Read Full License 


\section{Abstract}

Hepatocellular carcinoma (HCC) is the fourth leading cause of cancer-related death worldwide. Despite advancements in HCC diagnosis and treatment, current staging systems lack specificity and can't easily predict patient survival. Researchers now report a new prognostic and diagnostic approach based on tracking genes that control iron metabolism in the body. Iron is critical for normal cell metabolism, growth, and proliferation. That goes extra for tumor cells, which have an increased demand for iron. But too much iron can lead to cell death-a process known as "ferroptosis"-which some researchers are harnessing to eliminate harmful cancer cells. In the current study, researchers used high-throughput sequencing to identify genes associated with iron metabolism and ferroptosis in patients with HCC. Based on four genes, patients could be divided into a low-risk group and a high-risk group with poorer overall survival and HCC samples could be distinguished from normal samples. Further experiments revealed that erastin, a known inducer of ferroptosis, could effectively inhibit the growth of HCC tumors in mice. With more work, researchers could understand how to better spot signs of HCC early and improve treatment for patients with HCC. 